\title{
GENERALIZED ABSOLUTE RIESZ SUMMABILITY OF INFINITE SERIES AND FOURIER SERIES
}

\section{BAĞDAGÜL KARTAL*}

Department of Mathematics, Erciyes University, 38039 Kayseri, Turkey

${ }^{*}$ Corresponding author: bagdagulkartal@erciyes.edu.tr

\begin{abstract}
In this paper, two known theorems dealing with $\left|\bar{N}, p_{n}\right|_{k}$ summability of infinite series and Fourier series have been generalized to $\varphi-\left|\bar{N}, p_{n} ; \beta\right|_{k}$ summability.
\end{abstract}

\section{INTRODUCTION}

A sequence $\left(A_{n}\right)$ is said to be $\delta$-quasi-monotone if $A_{n} \rightarrow 0, A_{n}>0$ ultimately and $\Delta A_{n} \geq-\delta_{n}$, where $\Delta A_{n}=A_{n}-A_{n+1}$ and $\delta=\left(\delta_{n}\right)$ is a sequence of positive numbers (see [1]). A sequence $\left(g_{n}\right)$ is said to be of bounded variation, denoted by $\left(g_{n}\right) \in \mathcal{B V}$, if $\sum_{n=1}^{\infty}\left|\Delta g_{n}\right|<\infty$. Let $\sum a_{n}$ be a given infinite series with the partial sums $\left(s_{n}\right)$. Let $\left(\varphi_{n}\right)$ be a sequence of positive real numbers. The series $\sum a_{n}$ is said to be summable $\varphi-\left|\bar{N}, p_{n} ; \beta\right|_{k}, k \geq 1$ and $\beta \geq 0$, if (see [22])

$$
\sum_{n=1}^{\infty} \varphi_{n}^{\beta k+k-1}\left|u_{n}-u_{n-1}\right|^{k}<\infty
$$

where $\left(p_{n}\right)$ is a sequence of positive numbers such that

$$
P_{n}=\sum_{v=0}^{n} p_{v} \rightarrow \infty \quad \text { as } \quad n \rightarrow \infty \quad\left(P_{-i}=p_{-i}=0, \quad i \geq 1\right)
$$

Received August $13^{\text {th }}, 2020$; accepted September $1^{\text {st }}, 2020$; published September $14^{\text {th }}, 2020$.

2010 Mathematics Subject Classification. 26D15, 40D15, 40F05, 40G99.

Key words and phrases. absolute summability; Fourier series; Hölder's inequality; infinite series; Minkowski's inequality; Riesz mean; summability factor.

(C)2020 Authors retain the copyrights of their papers, and all open access articles are distributed under the terms of the Creative Commons Attribution License. 
and

$$
u_{n}=\frac{1}{P_{n}} \sum_{v=0}^{n} p_{v} s_{v}
$$

For $\varphi_{n}=\frac{P_{n}}{p_{n}}$ and $\beta=0, \varphi-\left|\bar{N}, p_{n} ; \beta\right|_{k}$ summability reduces to $\left|\bar{N}, p_{n}\right|_{k}$ summability (see [2]). Taking $\varphi_{n}=n, \beta=0$ and $p_{n}=1$ for all values of $n, \varphi-\left|\bar{N}, p_{n} ; \beta\right|_{k}$ summability reduces to $|C, 1|_{k}$ summability (see [8]).

If we write $X_{n}=\sum_{v=1}^{n} p_{v} / P_{v}$, then $\left(X_{n}\right)$ is a positive increasing sequence tending to infinity with $n$.

In [3], the following theorem on $\delta$-quasi-monotone sequences has been proved.

Theorem 1.1. Let $\left(\lambda_{n}\right) \rightarrow 0$ as $n \rightarrow \infty$ and $\left(p_{n}\right)$ be a sequence of positive numbers such that $P_{n}=O\left(n p_{n}\right)$ as $n \rightarrow \infty$. Suppose that there exists a sequence of numbers $\left(A_{n}\right)$ which is $\delta$-quasi-monotone with $\sum n X_{n} \delta_{n}<\infty, \sum A_{n} X_{n}$ is convergent, and $\left|\Delta \lambda_{n}\right| \leq\left|A_{n}\right|$ for all $n$. If the condition

$$
\sum_{n=1}^{m} \frac{p_{n}}{P_{n}}\left|t_{n}\right|^{k}=O\left(X_{m}\right) \quad \text { as } \quad m \rightarrow \infty
$$

is satisfied, where $\left(t_{n}\right)$ is the $n$-th $(C, 1)$ mean of the sequence $\left(n a_{n}\right)$, then the series $\sum a_{n} \lambda_{n}$ is summable $\left|\bar{N}, p_{n}\right|_{k}, k \geq 1$.

Lemma 1.1. [3] Under the conditions of Theorem 1.1, we have that

$$
\begin{gathered}
\left|\lambda_{n}\right| X_{n}=O(1) \text { as } n \rightarrow \infty, \\
n X_{n} A_{n}=O(1) \text { as } n \rightarrow \infty, \\
\sum_{n=1}^{\infty} n X_{n}\left|\Delta A_{n}\right|<\infty .
\end{gathered}
$$

\section{Main Result}

There are some papers on absolute summability (see $[4-6,9-12,16-18,23-25])$. Now we generalize Theorem 1.1 as in the following form.

Theorem 2.1. Let $\left(\varphi_{n}\right)$ be a sequence of positive real numbers such that

$$
\begin{gathered}
\varphi_{n} p_{n}=O\left(P_{n}\right), \\
\sum_{n=v+1}^{m+1} \varphi_{n}^{\beta k-1} \frac{1}{P_{n-1}}=O\left(\varphi_{v}^{\beta k} \frac{1}{P_{v}}\right) \quad \text { as } \quad m \rightarrow \infty .
\end{gathered}
$$


If all conditions of Theorem 1.1 are satisfied with the condition (1.1) replaced by

$$
\sum_{n=1}^{m} \varphi_{n}^{\beta k-1}\left|t_{n}\right|^{k}=O\left(X_{m}\right) \quad \text { as } \quad m \rightarrow \infty
$$

then the series $\sum a_{n} \lambda_{n}$ is summable $\varphi-\left|\bar{N}, p_{n} ; \beta\right|_{k}, k \geq 1$ and $0 \leq \beta<1 / k$.

\section{Proof of Theorem 2.1}

Let $\left(I_{n}\right)$ indicates $\left(\bar{N}, p_{n}\right)$ mean of the series $\sum a_{n} \lambda_{n}$. Then, for $n \geq 1$, we obtain

$$
\bar{\Delta} I_{n}=I_{n}-I_{n-1}=\frac{p_{n}}{P_{n} P_{n-1}} \sum_{v=1}^{n} P_{v-1} a_{v} \lambda_{v}=\frac{p_{n}}{P_{n} P_{n-1}} \sum_{v=1}^{n} \frac{P_{v-1} \lambda_{v}}{v} v a_{v} .
$$

Applying Abel's transformation, we get

$$
\begin{aligned}
\bar{\Delta} I_{n}= & \frac{p_{n}}{P_{n} P_{n-1}} \sum_{v=1}^{n-1} \frac{\lambda_{v+1}}{v} P_{v} t_{v}-\frac{p_{n}}{P_{n} P_{n-1}} \sum_{v=1}^{n-1} \frac{v+1}{v} p_{v} \lambda_{v} t_{v} \\
& +\frac{p_{n}}{P_{n} P_{n-1}} \sum_{v=1}^{n-1} \frac{v+1}{v} P_{v} t_{v} \Delta \lambda_{v}+\frac{(n+1)}{n P_{n}} p_{n} \lambda_{n} t_{n} \\
= & I_{n, 1}+I_{n, 2}+I_{n, 3}+I_{n, 4} .
\end{aligned}
$$

For the proof of Theorem 2.1, it is sufficient to show that

$$
\sum_{n=1}^{\infty} \varphi_{n}^{\beta k+k-1}\left|I_{n, r}\right|^{k}<\infty, \quad \text { for } \quad r=1,2,3,4
$$

First,

$$
\begin{aligned}
\sum_{n=2}^{m+1} \varphi_{n}^{\beta k+k-1}\left|I_{n, 1}\right|^{k} & \leq \sum_{n=2}^{m+1} \varphi_{n}^{\beta k+k-1}\left(\frac{p_{n}}{P_{n} P_{n-1}}\right)^{k}\left(\sum_{v=1}^{n-1} P_{v}\left|t_{v}\right| \frac{\left|\lambda_{v+1}\right|}{v}\right)^{k} \\
& =\sum_{n=2}^{m+1} \varphi_{n}^{\beta k-1}\left(\frac{\varphi_{n} p_{n}}{P_{n}}\right)^{k} \frac{1}{P_{n-1}^{k}}\left(\sum_{v=1}^{n-1} P_{v}\left|t_{v}\right| \frac{\left|\lambda_{v+1}\right|}{v}\right)^{k}
\end{aligned}
$$

Here (2.1) gives $\left(\frac{\varphi_{n} p_{n}}{P_{n}}\right)^{k}=O(1)$, also using Hölder's inequality, we obtain

$$
\sum_{n=2}^{m+1} \varphi_{n}^{\beta k+k-1}\left|I_{n, 1}\right|^{k}=O(1) \sum_{n=2}^{m+1} \varphi_{n}^{\beta k-1} \frac{1}{P_{n-1}}\left(\sum_{v=1}^{n-1} P_{v}\left|t_{v}\right|^{k} \frac{\left|\lambda_{v+1}\right|^{k}}{v}\right)\left(\frac{1}{P_{n-1}} \sum_{v=1}^{n-1} \frac{P_{v}}{v}\right)^{k-1}
$$

Now using the fact that $P_{v}=O\left(v p_{v}\right)$,

$$
\sum_{n=2}^{m+1} \varphi_{n}^{\beta k+k-1}\left|I_{n, 1}\right|^{k}=O(1) \sum_{n=2}^{m+1} \varphi_{n}^{\beta k-1} \frac{1}{P_{n-1}}\left(\sum_{v=1}^{n-1} p_{v}\left|t_{v}\right|^{k}\left|\lambda_{v+1}\right|^{k}\right)\left(\frac{1}{P_{n-1}} \sum_{v=1}^{n-1} p_{v}\right)^{k-1} .
$$


Then, we have

$$
\begin{aligned}
\sum_{n=2}^{m+1} \varphi_{n}^{\beta k+k-1}\left|I_{n, 1}\right|^{k} & =O(1) \sum_{n=2}^{m+1} \varphi_{n}^{\beta k-1} \frac{1}{P_{n-1}} \sum_{v=1}^{n-1} p_{v}\left|t_{v}\right|^{k}\left|\lambda_{v+1}\right|^{k} \\
& =O(1) \sum_{v=1}^{m} p_{v}\left|\lambda_{v+1}\right|^{k-1}\left|\lambda_{v+1}\right|\left|t_{v}\right|^{k} \sum_{n=v+1}^{m+1} \varphi_{n}^{\beta k-1} \frac{1}{P_{n-1}}
\end{aligned}
$$

Here, by using (2.2) and (1.2),

$$
\begin{aligned}
\sum_{n=2}^{m+1} \varphi_{n}^{\beta k+k-1}\left|I_{n, 1}\right|^{k} & =O(1) \sum_{v=1}^{m} \varphi_{v}^{\beta k} \frac{p_{v}}{P_{v}}\left|\lambda_{v+1}\right|\left|t_{v}\right|^{k} \\
& =O(1) \sum_{v=1}^{m} \varphi_{v}^{\beta k-1}\left(\frac{\varphi_{v} p_{v}}{P_{v}}\right)\left|\lambda_{v+1}\right|\left|t_{v}\right|^{k}
\end{aligned}
$$

Again, from (2.1), we obtain

$$
\sum_{n=2}^{m+1} \varphi_{n}^{\beta k+k-1}\left|I_{n, 1}\right|^{k}=O(1) \sum_{v=1}^{m} \varphi_{v}^{\beta k-1}\left|\lambda_{v+1}\right|\left|t_{v}\right|^{k} .
$$

Hence, we get

$$
\begin{aligned}
\sum_{n=2}^{m+1} \varphi_{n}^{\beta k+k-1}\left|I_{n, 1}\right|^{k} & =O(1) \sum_{v=1}^{m-1} \Delta\left|\lambda_{v+1}\right| \sum_{r=1}^{v} \varphi_{r}^{\beta k-1}\left|t_{r}\right|^{k}+O(1)\left|\lambda_{m+1}\right| \sum_{v=1}^{m} \varphi_{v}^{\beta k-1}\left|t_{v}\right|^{k} \\
& =O(1) \sum_{v=1}^{m-1}\left|A_{v+1}\right| X_{v+1}+O(1)\left|\lambda_{m+1}\right| X_{m+1}=O(1) \quad \text { as } \quad m \rightarrow \infty,
\end{aligned}
$$

by using Abel's transformation, hypotheses of Theorem 2.1, and Lemma 1.1.

Now, we have

$$
\begin{aligned}
\sum_{n=2}^{m+1} \varphi_{n}^{\beta k+k-1}\left|I_{n, 2}\right|^{k} & =O(1) \sum_{n=2}^{m+1} \varphi_{n}^{\beta k+k-1}\left(\frac{p_{n}}{P_{n} P_{n-1}}\right)^{k}\left(\sum_{v=1}^{n-1} p_{v}\left|\lambda_{v}\right|\left|t_{v}\right|\right)^{k} \\
& =O(1) \sum_{n=2}^{m+1} \varphi_{n}^{\beta k-1}\left(\frac{\varphi_{n} p_{n}}{P_{n}}\right)^{k} \frac{1}{P_{n-1}^{k}}\left(\sum_{v=1}^{n-1} p_{v}\left|\lambda_{v}\right|\left|t_{v}\right|\right)^{k} \\
& =O(1) \sum_{n=2}^{m+1} \varphi_{n}^{\beta k-1} \frac{1}{P_{n-1}^{k}}\left(\sum_{v=1}^{n-1} p_{v}\left|\lambda_{v}\right|\left|t_{v}\right|\right)^{k} .
\end{aligned}
$$

Using Hölder's inequality, we get

$$
\begin{aligned}
\sum_{n=2}^{m+1} \varphi_{n}^{\beta k+k-1}\left|I_{n, 2}\right|^{k} & =O(1) \sum_{n=2}^{m+1} \varphi_{n}^{\beta k-1} \frac{1}{P_{n-1}}\left(\sum_{v=1}^{n-1} p_{v}\left|\lambda_{v}\right|^{k}\left|t_{v}\right|^{k}\right)\left(\frac{1}{P_{n-1}} \sum_{v=1}^{n-1} p_{v}\right)^{k-1} \\
& =O(1) \sum_{v=1}^{m} p_{v}\left|\lambda_{v}\right|^{k}\left|t_{v}\right|^{k} \sum_{n=v+1}^{m+1} \varphi_{n}^{\beta k-1} \frac{1}{P_{n-1}}
\end{aligned}
$$


By (2.2), (2.1) and (1.2), we get

$$
\sum_{n=2}^{m+1} \varphi_{n}^{\beta k+k-1}\left|I_{n, 2}\right|^{k}=O(1) \sum_{v=1}^{m} \varphi_{v}^{\beta k-1}\left|\lambda_{v}\right|\left|t_{v}\right|^{k}
$$

Here, using Abel's transformation as in $I_{n, 1}$, we have

$$
\sum_{n=2}^{m+1} \varphi_{n}^{\beta k+k-1}\left|I_{n, 2}\right|^{k}=O(1) \text { as } m \rightarrow \infty
$$

Again, using Hölder's inequality, we have

$$
\begin{aligned}
\sum_{n=2}^{m+1} \varphi_{n}^{\beta k+k-1}\left|I_{n, 3}\right|^{k} & =O(1) \sum_{n=2}^{m+1} \varphi_{n}^{\beta k+k-1}\left(\frac{p_{n}}{P_{n} P_{n-1}}\right)^{k}\left(\sum_{v=1}^{n-1} P_{v}\left|t_{v}\right|\left|\Delta \lambda_{v}\right|\right)^{k} \\
& =O(1) \sum_{n=2}^{m+1} \varphi_{n}^{\beta k-1}\left(\frac{\varphi_{n} p_{n}}{P_{n}}\right)^{k} \frac{1}{P_{n-1}^{k}}\left(\sum_{v=1}^{n-1} P_{v}\left|t_{v}\right|\left|A_{v}\right|\right)^{k} \\
& =O(1) \sum_{n=2}^{m+1} \varphi_{n}^{\beta k-1} \frac{1}{P_{n-1}}\left(\sum_{v=1}^{n-1} p_{v}\left|t_{v}\right|^{k}\left(v\left|A_{v}\right|\right)^{k}\right)\left(\frac{1}{P_{n-1}} \sum_{v=1}^{n-1} p_{v}\right)^{k-1} \\
& =O(1) \sum_{n=2}^{m+1} \varphi_{n}^{\beta k-1} \frac{1}{P_{n-1}} \sum_{v=1}^{n-1} p_{v}\left|t_{v}\right|^{k}\left(v\left|A_{v}\right|\right)^{k-1}\left(v\left|A_{v}\right|\right)
\end{aligned}
$$

Using (1.3), we get $\left(v\left|A_{v}\right|\right)^{k-1}=O(1)$, then

$$
\sum_{n=2}^{m+1} \varphi_{n}^{\beta k+k-1}\left|I_{n, 3}\right|^{k}=O(1) \sum_{v=1}^{m} p_{v}\left|t_{v}\right|^{k} v\left|A_{v}\right| \sum_{n=v+1}^{m+1} \varphi_{n}^{\beta k-1} \frac{1}{P_{n-1}}
$$

Now using the conditions (2.2) and (2.1), we get

$$
\sum_{n=2}^{m+1} \varphi_{n}^{\beta k+k-1}\left|I_{n, 3}\right|^{k}=O(1) \sum_{v=1}^{m} \varphi_{v}^{\beta k-1}\left|t_{v}\right|^{k} v\left|A_{v}\right|
$$

Then, we have

$$
\begin{aligned}
\sum_{n=2}^{m+1} \varphi_{n}^{\beta k+k-1}\left|I_{n, 3}\right|^{k} & =O(1) \sum_{v=1}^{m-1} \Delta\left(v\left|A_{v}\right|\right) \sum_{r=1}^{v} \varphi_{r}^{\beta k-1}\left|t_{r}\right|^{k}+O(1) m\left|A_{m}\right| \sum_{v=1}^{m} \varphi_{v}^{\beta k-1}\left|t_{v}\right|^{k} \\
& =O(1) \sum_{v=1}^{m-1} \Delta\left(v\left|A_{v}\right|\right) X_{v}+O(1) m\left|A_{m}\right| X_{m} \\
& =O(1) \sum_{v=1}^{m-1} v X_{v}\left|\Delta A_{v}\right|+O(1) \sum_{v=1}^{m-1}\left|A_{v+1}\right| X_{v+1}+O(1) m\left|A_{m}\right| X_{m} \\
& =O(1) \text { as } m \rightarrow \infty
\end{aligned}
$$

by using Abel's transformation, hypotheses of Theorem 2.1, and Lemma 1.1. 
Finally, we get

$$
\begin{aligned}
\sum_{n=1}^{m} \varphi_{n}^{\beta k+k-1}\left|I_{n, 4}\right|^{k} & =O(1) \sum_{n=1}^{m} \varphi_{n}^{\beta k+k-1}\left(\frac{p_{n}}{P_{n}}\right)^{k}\left|\lambda_{n}\right|^{k-1}\left|\lambda_{n}\right|\left|t_{n}\right|^{k} \\
& =O(1) \sum_{n=1}^{m} \varphi_{n}^{\beta k-1}\left|\lambda_{n}\right|\left|t_{n}\right|^{k}
\end{aligned}
$$

Here, as in $I_{n, 1}$, we get

$$
\sum_{n=1}^{m} \varphi_{n}^{\beta k+k-1}\left|I_{n, 4}\right|^{k}=O(1) \text { as } m \rightarrow \infty .
$$

Hence, the proof of Theorem 2.1 is completed.

\section{Applications}

There are some different papers dealing with applications of Fourier series (see [14,15,19-21]). Let $f$ be a periodic function with period $2 \pi$ and Lebesgue integrable over $(-\pi, \pi)$. The trigonometric Fourier series of $f$ is defined as

$$
f(x) \sim \frac{1}{2} a_{0}+\sum_{n=1}^{\infty}\left(a_{n} \cos n x+b_{n} \sin n x\right)=\sum_{n=0}^{\infty} C_{n}(x)
$$

where

$$
a_{0}=\frac{1}{\pi} \int_{-\pi}^{\pi} f(x) d x, \quad a_{n}=\frac{1}{\pi} \int_{-\pi}^{\pi} f(x) \cos (n x) d x \quad \text { and } \quad b_{n}=\frac{1}{\pi} \int_{-\pi}^{\pi} f(x) \sin (n x) d x .
$$

Write

$$
\phi(t)=\frac{1}{2}\{f(x+t)+f(x-t)\}
$$

and

$$
\phi_{1}(t)=\frac{1}{t} \int_{0}^{t} \phi(u) d u
$$

If $\phi_{1}(t) \in \mathcal{B V}(0, \pi)$, then $t_{n}(x)=O(1)$, where $t_{n}(x)$ is the $\mathrm{n}$-th $(C, 1)$ mean of the sequence $\left(n C_{n}(x)\right)$ (see [7]). By using this, the following theorem has been obtained in [3].

Theorem 4.1. If $\phi_{1}(t) \in \mathcal{B V}(0, \pi)$, and the sequences $\left(p_{n}\right),\left(\lambda_{n}\right)$ and $\left(X_{n}\right)$ satisfy the conditions of Theorem 1.1 , then the series $\sum C_{n}(x) \lambda_{n}$ is summable $\left|\bar{N}, p_{n}\right|_{k}, k \geq 1$.

The following theorem gives a generalization of Theorem 4.1 for $\varphi-\left|\bar{N}, p_{n} ; \beta\right|_{k}$ summability.

Theorem 4.2. If $\phi_{1}(t) \in \mathcal{B} \mathcal{V}(0, \pi)$, and the sequences $\left(p_{n}\right),\left(\lambda_{n}\right),\left(A_{n}\right),\left(\varphi_{n}\right)$ and $\left(X_{n}\right)$ satisfy the conditions of Theorem 2.1, then the series $\sum C_{n}(x) \lambda_{n}$ is summable $\varphi-\left|\bar{N}, p_{n} ; \beta\right|_{k}, k \geq 1$ and $0 \leq \beta<1 / k$. 


\section{Conclusions}

If we take $\varphi_{n}=\frac{P_{n}}{p_{n}}$ and $\beta=0$ in Theorem 2.1, then the condition (2.3) reduces to the condition (1.1), and the conditions (2.1) and (2.2) are provided. Thus, Theorem 2.1 reduces to Theorem 1.1. If we take $\varphi_{n}=n, \beta=0$ and $p_{n}=1$ for all values of $n$, then we have a result for $|C, 1|_{k}$ summability of an infinite series (see [13]). Also, if we take $\varphi_{n}=\frac{P_{n}}{p_{n}}$ and $\beta=0$ in Theorem 4.2, then we get Theorem 4.1.

Conflicts of Interest: The author(s) declare that there are no conflicts of interest regarding the publication of this paper.

\section{REFERENCES}

[1] R.P. Boas, Quasi-positive sequences and trigonometric series, Proc. Lond. Math. Soc. s3-14A (1965), 38-46.

[2] H. Bor, On two summability methods, Math. Proc. Cambridge Philos. Soc. 97 (1) (1985), 147-149.

[3] H. Bor, On quasi-monotone sequences and their applications, Bull. Austral. Math. Soc. 43 (2) (1991), 187-192.

[4] H. Bor, H. S. Özarslan, On absolute Riesz summability factors, J. Math. Anal. Appl. 246 (2) (2000), 657-663.

[5] H. Bor, H. S. Özarslan, A note on absolute summability factors, Adv. Stud. Contemp. Math. (Kyungshang) 6 (1) (2003), $1-11$.

[6] H. Bor, H. Seyhan, On almost increasing sequences and its applications, Indian J. Pure Appl. Math. 30 (10) (1999), 1041-1046.

[7] K.K. Chen, Functions of bounded variation and the Cesàro means of a Fourier series, Acad. Sinica Science Record 1 (1945), 283-289.

[8] T.M. Flett, On an extension of absolute summability and some theorems of Littlewood and Paley, Proc. Lond. Math. Soc. s3-7 (1957), 113-141.

[9] A. Karakaş, A note on absolute summability method involving almost increasing and $\delta$-quasi-monotone sequences, Int. J. Math. Comput. Sci. 13 (1) (2018), 73-81.

[10] A. Karakaş, A new factor theorem for generalized absolute Riesz summability, Carpathian Math. Publ. 11 (2) (2019), 345-349.

[11] B. Kartal, On generalized absolute Riesz summability method, Commun. Math. Appl. 8 (3) (2017), 359-364.

[12] B. Kartal, New results for almost increasing sequences, Ann. Univ. Paedagog. Crac. Stud. Math. 18 (2019), 85-91.

[13] S.M. Mazhar, On generalized quasi-convex sequence and its applications, Indian J. Pure Appl. Math. 8 (7) (1977), 784-790.

[14] H.S. Özarslan, A note on $\left|\bar{N}, p_{n} ; \delta\right|_{k}$ summability factors, Erc. Üni. Fen Bil. Enst. Derg., cilt. 16 (2000), 95-100.

[15] H.S. Özarslan, A note on $\left|\bar{N}, p_{n}^{\alpha}\right|_{k}$ summability factors, Soochow J. Math. 27 (1) (2001), 45-51.

[16] H.S. Özarslan, On almost increasing sequences and its applications, Int. J. Math. Math. Sci. 25 (5) (2001), $293-298$.

[17] H.S. Özarslan, A note on $\left|\bar{N}, p_{n} ; \delta\right|_{k}$ summability factors, Indian J. Pure Appl. Math. 33 (3) (2002), 361-366.

[18] H.S. Özarslan, On $\left|\bar{N}, p_{n} ; \delta\right|_{k}$ summability factors, Kyungpook Math. J. 43 (1) (2003), 107-112.

[19] H.S. Özarslan, A note on $\left|\bar{N}, p_{n}\right|_{k}$ summability factors, Int. J. Pure Appl. Math. 13 (4) (2004), 485-490.

[20] H.S. Özarslan, On the local properties of factored Fourier series, Proc. Jangjeon Math. Soc. 9 (2) (2006), 103-108.

[21] H.S. Özarslan, Local properties of factored Fourier series, Int. J. Comp. Appl. Math. 1 (1) (2006), 93-96.

[22] H. Seyhan, On the local property of $\varphi-\left|\bar{N}, p_{n} ; \delta\right|_{k}$ summability of factored Fourier series, Bull. Inst. Math. Acad. Sin. 25 (4) (1997), 311-316. 
[23] H. Seyhan, A note on absolute summability factors, Far East J. Math. Sci. 6 (1) (1998), 157-162.

[24] H. Seyhan, On the absolute summability factors of type (A,B), Tamkang J. Math. 30 (1) (1999), 59-62.

[25] H. Seyhan, A. Sönmez, On $\varphi-\left|\bar{N}, p_{n} ; \delta\right|_{k}$ summability factors, Portugal. Math. 54 (4) (1997), 393-398. 